\title{
Complex treatment of progressive proliferative diabetic retinopathy with concomitant diabetic macular edema - a case report
}

\author{
Agnieszka Kalinowska', Beata Gajda-Deryło ${ }^{1}$, Piotr kukasik², Anna Rejman², \\ Katarzyna Nowomiejska ${ }^{1}$, Robert Rejdak ${ }^{1}$ \\ 'Department of General Ophthalmology, Medical University of Lublin, Poland \\ ${ }^{2}$ Department of Ophthalmology, Zamosc, Poland
}

\begin{abstract}
Proliferative diabetic retinopathy (PDR) is a major cause of visual impairment in working — age adult population worldwide. The purpose of this article is to report the long observation and treatment of a patient with severe PDR. We present a case of 42 years old female who had been diagnosed with diabetes mellitus type 1 thirteen years ago, presenting a progressive PDR and macular edema in both eyes. The patient was monitored during three years of the follow-up. The treatment included laser photocoagulation, anty-VEGF injections and the surgical treatment including vitrectomy with ILM peeling and gas or silicone oil tamponade.
\end{abstract}

KEY WORDS: proliferative diabetic retinopathy; diabetic macular edema; anty-VEGF drugs; vitrectomy

Ophthalmol J 2018; Vol. 3, No. 2, 68-72

\section{INTRODUCTION}

Diabetes mellitus (DM) affected > 350 million people worldwide in 2013, and the number is estimated to increase to $>590$ million people by 2035 [1]. Over one-third of diabetic patients have signs of DR and a third of these are affected with vision-threatening diabetic retinopathy (VTDR), defined as severe non-proliferative diabetic retinopathy (DR) or proliferative DR (PDR) or the presence of diabetic macular edema (DME) [2]. Estimates on DR prevalence in type 1 diabetes in Europe and the USA range between 36.5-93.6\%, with VTDR prevalence estimated between $6.7-34.9 \%$ [2].

The gold standard treatment for PDR includes panretinal photocoagulation (PRP) [3] PRP was initiated as a treatment option in the 1960s and was documented as beneficial over time. It is used to improve the retinal circulation, allowing more oxygen to reach the retina and limit- ing the formation and release of pro-angiogenic cytokines [4]. Adverse effects of PRP as loss of peripheral visual field, worsening of traction, problems with performing this procedure in eyes with media opacity and pain during laser treatment have prompted research into alternative therapy for PDR eyes. The elevated intra-ocular levels of pro-angiogenic factors, especially vascular endothelial growth factor (VEGF) in the pathophysiology of PDR gives the background to use anti-VEGF injections to treat and proved to be a promising potential alternative.

Intravitreal injections of VEGF inhibitors are currently the standard treatment for DME. DME affects $-30 \%$ of patients with DR and is a major factor leading to vision loss [5]. It can occur at any stage of DR, and the risk increases with the severity of the disease: $3 \%$ cases with mild non-proliferative diabetic retinopathy (NPDR), 38\% cases 
with moderate-to-severe NPDR, and $71 \%$ cases with PDR [6].

Surgery for diabetic retinopathy addresses late secondary complications. Typical indications for vitrectomy are vitreous haemorrhage, tractional retinal detachment, combined tractional-rhegmatogenous retinal detachment, and severe fibrovascular proliferation (including macular distortion or dragging, tractional macular oedema). These vitreoretinal conditions have been treated with increasingly successful outcomes since the introduction of pars plana vitrectomy in the early 1970s. [7].

We report a case of a patient with severe, rapidly progressive proliferative diabetic retinopathy in both eyes with concomitant macular edema, requiring complex therapy to achieve the best visual outcomes.

\section{CASE REPORT}

A forty-two years old female presented in our retinal out-patient clinic in February 2015 with complains of blurred vision in her left eye from two months. Her past medical history revealed 13 years lasting insulin dependent diabetes mellitus, she had undergone appropriate treatment with levels of $\mathrm{HbA}_{1 \mathrm{c}}$ around $8.0 \%$.

At first visit her best-corrected visual acuity was on the right eye 1.0 and on the left eye 0.4 with Snellen decimal charts. Clinical examination of the anterior segment showed early opacifications of the lens. Dilated fundus examination revealed the early stage of PDR in the right eye (Fig. 1) and advanced

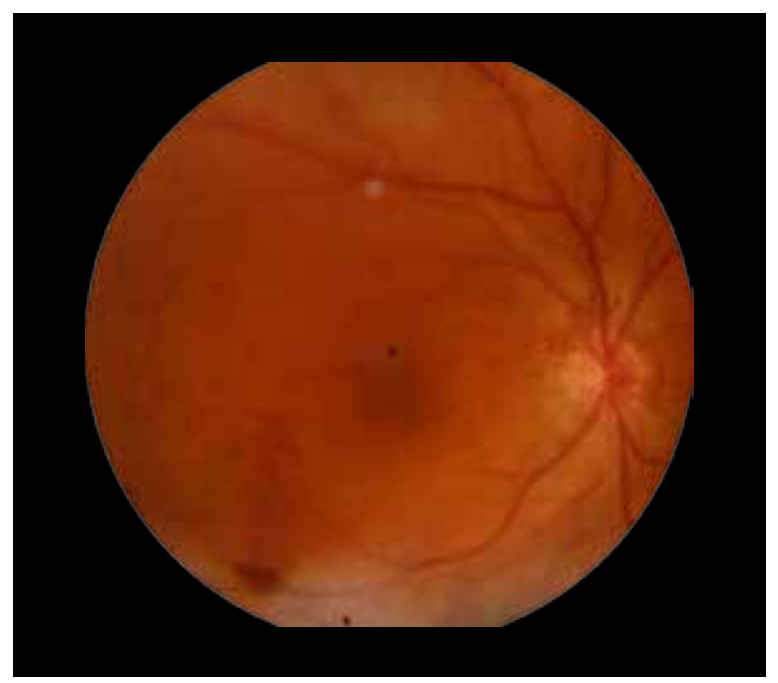

FIGURE 1. Neovascularization on the optic nerve disc of the right eye

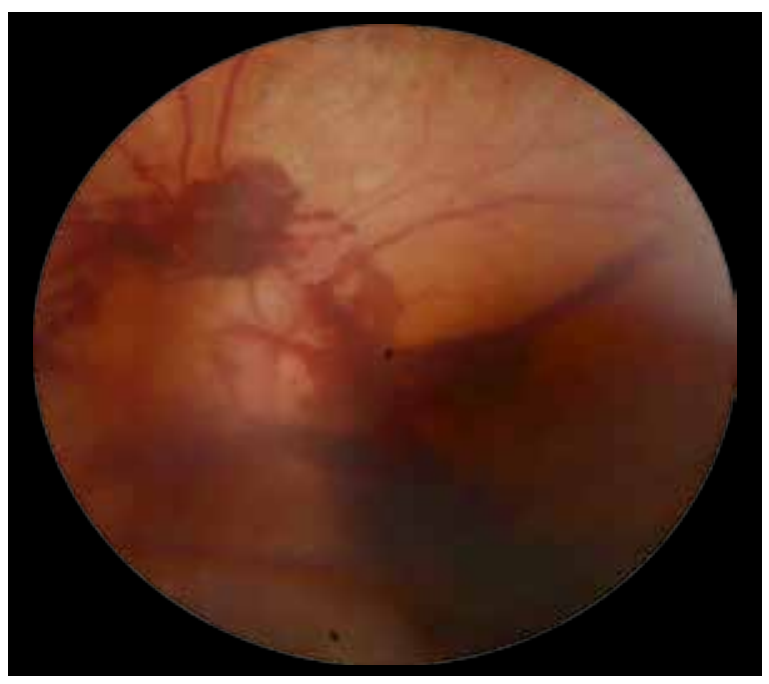

FIGURE 2. Vitreous haemorrhage due to neovascularisation at the optic disc of the left eye

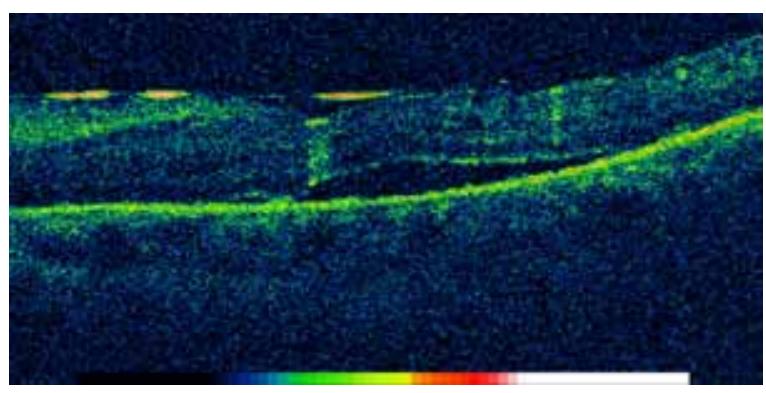

FIGURE 3. Tractional retinal detachment involving macula due to extensive fibrovascular membranes in the left eye

PDR changes with clinically significant $\mathrm{ME}$ in the left eye (Fig. 2). The initial treatment consisted of three sessions of PFC because of neovascularization due to non-perfused areas of the retina. After this time, further laser treatment was impossible because of the expanding cataract. The best corrected visual acuity was on the right eye 0.5 and on the left eye 0.1 . Cataract surgery (phacoemulsification and intraocular lens implantation) was performed on the patient's both eyes. At the first control after cataract surgery the best corrected visual acuity in right eye improved to 0.7 (Snellen) and on the left eye to 0.8 (Snellen). But after 2 weeks from surgery, the vision of left eye significantly deteriorated and the vitreous haemorrhage with tractional retinal detachment involving the macula was diagnosed (Fig. 3). Pars plana vitrectomy with removal of fibrovascular membranes and ILM peeling and silicone oil tamponade was performed after one month (Fig. 4). Five days before the surgery anti-VEGF injection of 


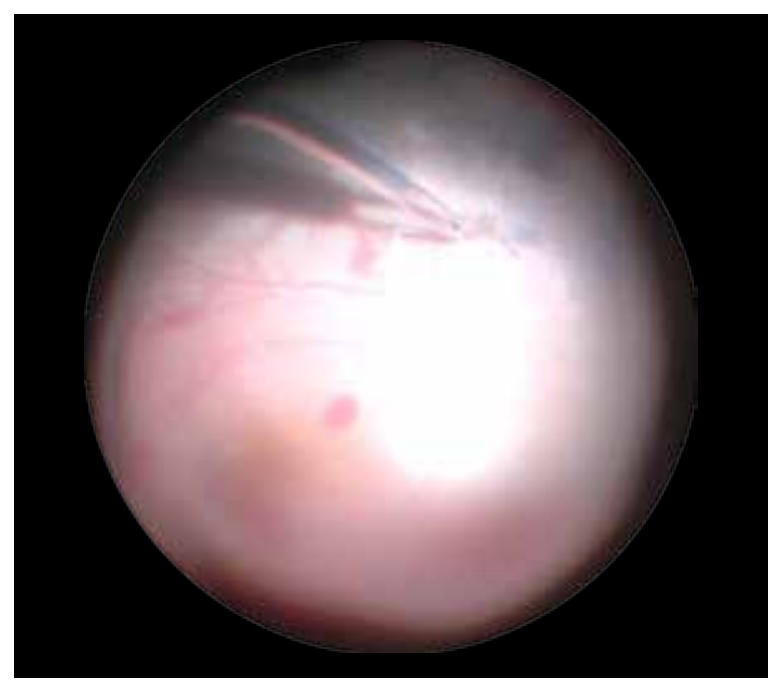

FIGURE 4. Vitrectomy with removing fibrovascular membanes across the posterior pole of the left eye

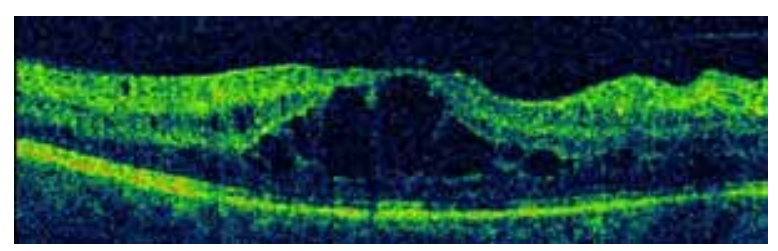

FIGURE 5. Diabetic macular edema before treatment of the right eye

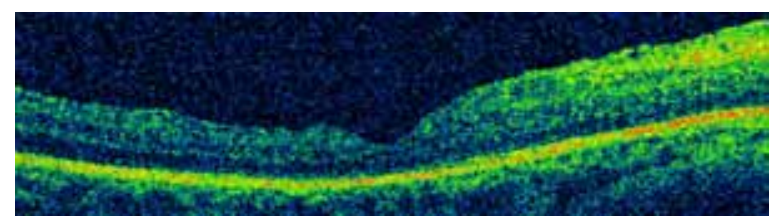

FIGURE 6. Macula after anty-VEGF injections and pars plana vitrectomy of the right eye

aflibercept was given to the left eye. The PFC was continued in both eyes.

Due to PDR and DME in the right eye, the patient was given five intravitreal injections of aflibercept through one year (Fig. 5, 6). After one year the second PPV with ILM peeling and removal of silicone oil was done in the left eye. Post-surgically the corrected visual acuity of the left eye improved to 0.3 and has been stable since then there was no need to treat the left eye.

Unfortunately, the condition of the right eye was getting worse, there was necessary to do another PFC session and Yag capsulotomy. The treatment was not sufficient, there was vitreous hemorrhage and deterioration of vision for hand movement.

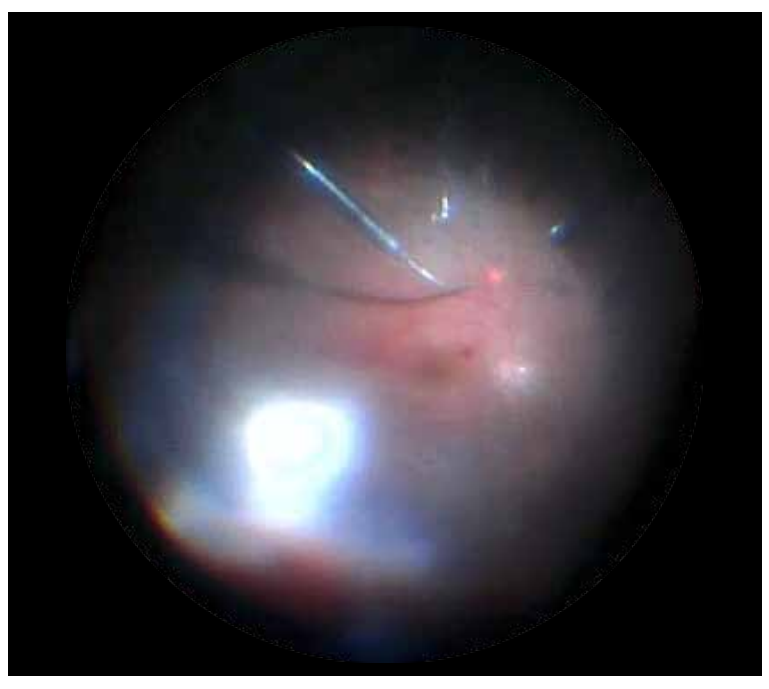

FIGURE 7. Intraoperative photocoagulation during vitrectomy of the right eye

Vitreous surgery with ILM peeling, vitreous haemorrhage removal and endolaser and gas tamponade was performed (Fig. 7). Five days before the surgery anti-VEGF injection of aflibercept was given to the right eye. After four months of observation, there is no $\mathrm{ME}$ and neovascularization in the right eye. The corrected visual acuity in the right eye improved to 0.4 .

\section{DISCUSSION}

In the present case report, we have shown the complex treatment of progressive DR in a patient with type $1 \mathrm{DM}$. Diabetic retinopathy (DR) is characterized by retinal microvascular damage, based on ischaemic changes and increased capillary permeability. The DR it is a complex multifactorial disease with many risk factors involved. The most obvious systemic risk factor responsible for developing DR is hyperglycemia. The result of chronic hyperglycemia is activation four main mechanisms that are responsible for the occurrence of the DR: increased polyol pathway flux, increased advanced glycation end-products (AGEs) formation, increased hexosamine pathway flux, and activation of protein kinase $\mathrm{C}$ (PKC). Improper operation each of this pathways leads to early apoptosis, progressive capillary occlusion, extracellular matrix overproduction, and deposition of plasma proteins. Furthermore, they are associated with overproduction of reactive oxygen species (ROS). These biochemical changes disturb the balance of pro-angiogenesis and antiangiogenesis factors involved in the progression of DR. Vascular 
endothelial growth factor (VEGF), is one of the bestknown angiogenic factors. The treatment of DMO is based on intravitreal anty-VEGF drugs and intravitreal corticosteroids, laser photocoagulation and in some cases, the vitreoretinal surgery can be helpful. For PDR, the gold standard treatment is laser PFC and vitreoretinal surgery, the intravitreal anty-VEGF drugs are also used especially in combination with PRP or before vitreoretinal surgery.

PFC has stood the test of time and is the only evidence-based treatment available for PDR. Studies have shown almost 50-60\% reduction in risk of sever visual loss with regression of neovascularization post laser treatment [3]. It is postulated that PRP decreases the signal for angiogenesis, promotes regression of neovascularization, and decreases the risk of hemorrhage or development of tractional membranes. VEGF levels in the vitreous were shown to decline after PRP, and regression of PDR was confirmed on the exam [8]. However, PRP has well-established limitations. It requires a cooperative patient and a clear view of the retina. Peripheral vision is permanently lost with the destruction of the peripheral retina, and nyctalopia and a transient decline in visual acuity (VA) have also been reported [9]. Macular edema can develop or worsen after PRP, adversely affecting vision and nearly $4.5 \%$ show disease progression that ultimately requires pars plana vitrectomy (PPV), even when PRP was considered "adequate". This has impelled the efforts to provide alternative treatment options that may delay or obviate the need for PRP.

In recent years, the development of molecules that target VEGF itself has changed the approach to diabetic retinopathy. There were a few important clinical trials of anti-VEGF for DME. The RISE/RIDE studies were two parallel randomized control trials that evaluated long-term visual outcomes in patients with DME who were treated solely with ranibizumab. The VIVID/VISTA studies compared intravitreal aflibercept, $2 \mathrm{mg}$, every 4 or 8 weeks with macular laser for DME. Protocol $\mathrm{T}$ comparison study of bevacizumab, ranibizumab, and aflibercept for DME. All of them showed statistically significant efficacy anti-VEGF in treatment DME. During the studies, investigators noted that eyes randomized to the anti-VEGF group were more likely to have regression of diabetic retinopathy and were less likely to develop PDR. DRCR Protocol S was a randomized clinical trial designed to determine whether ranibizumab, $0.5 \mathrm{mg}$, was similar to PRP for PDR. In Protocol S, ranibizumab-treated eyes had better VA outcomes, less development of DME, and less need for vitrectomy. In eyes with PDR and vitreous haemorrhage that makes impossible to do PRP, anti-VEGF treatment can be used to treat the underlying retinopathy and may delay but not reduce the need for surgery. DRCR Protocol $\mathrm{H}$ did not show a significant difference in rates of vitrectomy at 16 weeks between saline injection or ranibizumab-injected eyes. Eyes that received ranibizumab did have better VA outcomes. In addition, anti-VEGF inhibiting angiogenesis may increase the concentration of connective tissue growth factor (CTGF) that promotes fibrosis. This, in turn, leads to increase vitreoretinal tractions and risk of tractional retinal detachment or haemorrhage [10].

Many reports have suggested that vitrectomy may be beneficial in the treatment of ME with vitreomacular traction [11] but also for diffuse DMO, even without any tractional component $[12,13]$. The vitreous gel contains several growth factors, including best known VEGF [8], during vitrectomy it is removed from the eye and the presence of them could be lower. Furthermore, vitrectomy with surgically induced posterior vitreous detachment may not only release any unobserved vitreous traction on the macula but may also eliminate the condensed fluid containing growth factors.

The Diabetic Retinopathy Vitrectomy Study (DRVS) defined the benefit of early vitrectomy in the management of severe vitreous haemorrhage. The greatest benefit was demonstrated for patients with type 1 diabetes who tended to have more severe PDR. The benefits of early vitrectomy remained after 4 years follow-up [14].

More recently it has been reported that intravitreal injection of bevacizumab 1 week preoperatively can reduce the risk of bleeding from pathological vessels during surgery and in the early post-operative period [15-16].

Prevention and treatment of diabetic retinopathy are multidirectional, treating a young patient with diabetic retinopathy is still a challenge. Long life perspective, high expectation, poor long- time compliance in young patients makes the treatment more difficult. Further analysis of DRCR protocol $\mathrm{T}$ and $\mathrm{S}$ also remind us that despite treatment, approximately $30 \%$ of eyes with retinopathy will continue to worsen.

\section{REFERENCES}

1. Kharroubi AT, Darwish HM. Diabetes mellitus: The epidemic of the century. World J Diabetes. 2015; 6(6): 850-867, doi: 10.4239/wjd. v6.i6.850, indexed in Pubmed: 26131326. 
2. Lee R, Wong TY, Sabanayagam C. Epidemiology of diabetic retinopathy, diabetic macular edema and related vision loss. Eye Vis (Lond). 2015; 2: 17, doi: 10.1186/s40662-015-0026-2, indexed in Pubmed: 26605370.

3. Photocoagulation treatment of proliferative diabetic retinopathy. Clinical application of Diabetic Retinopathy Study (DRS) findings, DRS Report Number 8. The Diabetic Retinopathy Study Research Group. Ophthalmology. 1981; 88(7): 583-600, indexed in Pubmed: 7196564.

4. Kinshuck D. Proliferative retinopathy. http://www.diabeticretinopathy. org.uk/proliferative.html\#I. (accessed January 14, 2018).

5. Dugel PU, Hillenkamp J, Sivaprasad S, et al. Baseline visual acuity strongly predicts visual acuity gain in patients with diabetic macular edema following anti-vascular endothelial growth factor treatment across trials. Clin Ophthalmol. 2016; 10: 1103-1110, doi: 10.2147/ OPTH.S100764, indexed in Pubmed: 27366049.

6. Diabetic Retinopathy with Diabetic Macular Edema. https://www. genentech-forum.com/content/dam/gene/managedcare/forum/pdfs/ Disease-Information/diabetic-retinopathy-with-diabetic-macularedema-disease-state-overview.pdf. (accessed January 14, 2018).

7. Machemer $R$, Buettner $H$, Norton EW, et al. Vitrectomy: a pars plana approach. Trans Am Acad Ophthalmol Otolaryngol. 1971; 75(4): 813-820, indexed in Pubmed: 5566980.

8. Aiello LP, Avery RL, Arrigg PG, et al. Vascular endothelial growth factor in ocular fluid of patients with diabetic retinopathy and other retinal disorders. N Engl J Med. 1994; 331(22): 1480-1487, doi: 10.1056/ NEJM199412013312203, indexed in Pubmed: 7526212.

9. Flynn HW, Chew EY, Simons BD, et al. Pars plana vitrectomy in the Early Treatment Diabetic Retinopathy Study. ETDRS report number 17. The Early Treatment Diabetic Retinopathy Study Research Group. Ophthalmology. 1992; 99(9): 1351-1357, indexed in Pubmed: 1407968.
10. Kuiper EJ, Van Nieuwenhoven FA, de Smet MD, et al. The angio-fibrotic switch of VEGF and CTGF in proliferative diabetic retinopathy. PLOS One. 2008; 3(7): e2675, doi: 10.1371/journal.pone.0002675, indexed in Pubmed: 18628999.

11. Kaiser P, Riemann C, Sears J, et al. Macular traction detachment and diabetic macular edema associated with posterior hyaloidal traction. Am J Ophthalmol. 2001; 131(1): 44-49, doi: 10.1016/s00029394(00)00872-2, indexed in Pubmed: 11162978.

12. Recchia FM, Ruby AJ, Carvalho Recchia CA. Pars plana vitrectomy with removal of the internal limiting membrane in the treatment of persistent diabetic macular edema. Am J Ophthalmol. 2005; 139(3): 447-454, doi: 10.1016/j.ajo.2004.09.076, indexed in Pubmed: 15767052.

13. Stolba U, Binder $S$, Gruber D, et al. Vitrectomy for persistent diffuse diabetic macular edema. Am J Ophthalmol. 2005; 140(2): 295-301, doi: 10.1016/j.ajo.2005.03.045, indexed in Pubmed: 16023067.

14. Early vitrectomy for severe vitreous hemorrhage in diabetic retinopathy. Four-year results of a randomized trial: Diabetic Retinopathy Vitrectomy Study Report 5. Arch Ophthalmol. 1990; 108(7): 958-964, doi: 10.1001/archopht.1990.01070090060040, indexed in Pubmed: 2196036.

15. Yang $\mathrm{CM}$, Yeh PT, Yang $\mathrm{CH}$, et al. Bevacizumab pretreatment and longacting gas infusion on vitreous clear-up after diabetic vitrectomy. Am J Ophthalmol. 2008; 146(2): 211-217, doi: 10.1016/..ajo.2008.04.028, indexed in Pubmed: 18547539.

16. Rizzo $S$, Genovesi-Ebert F, Di Bartolo $E$, et al. Injection of intravitreal bevacizumab (Avastin) as a preoperative adjunct before vitrectomy surgery in the treatment of severe proliferative diabetic retinopathy (PDR). Graefes Arch Clin Exp Ophthalmol. 2008; 246(6): 837-842, doi: 10.1007/s00417-008-0774-y, indexed in Pubmed: 18286296. 